\title{
Aislamiento de especies termotolerantes de Campylobacter en dos poblaciones de pollos criados con y sin confinamiento*
}

\author{
Isolation of thermotolerant Campylobacter species from two populations of \\ chickens bred in confinement and at liberty
}

\author{
Alvaro Tresierra-Ayala, Heriberto Fernández, Maria E. Bendayán, Gustavo Pereyra, Alfonso Bernuy \\ Departamento de Microbiologla. Universidad Nacional de la Amazonia Peruana - Perú \\ (A.T.A, M.E.B., A.B.), \\ Instituto de Microbiología Clínica. Universidad Austral de Chile - Valdivia - Chile (H.F.)
}

\begin{abstract}
Se estudió la frecuencia del aislamiento de Campylobacter spp. en pollos domésticos y pollos mantenidos en confinamiento permanente, en la ciudad de lquitos (Perú). Campylobacterspp. fue aislado en $54,0 \%$ en el primer grupo y $35,0 \%$ en el segundo ( $p<0,05$ ). De las especies termotolerantes clásicas, las más frecuentes fueron $C$. jejuni y C. coli. La presencia de $C$. lari en estas aves, señala la importancia de ellas como reservorio natural de este microorganismo.
\end{abstract}

Campylobacter, isolación. Pollos, microbiología. Reservorio de enfermedades.

\section{Introducclón}

En los últimos años, las especies termotolerantes de Campylobacter han emergido como una causa común de diarrea en humanos ${ }^{4,5,11}$. Estos microorganismos, ampliamente distribuidos en el mundo, tienen como reservorios naturales una gran variedad de especies animales, tanto domésticas como de vida libre, siendo las aves domésticas una de las principales fuentes de infección para el hombre ${ }^{1,3,4,9,111,13,16,19,21.23}$.

En Iquitos(Amazonía Peruana, $3^{\circ} 45^{\prime} 05^{\prime \prime}$ lat. sur), un alto porcentaje $(23,0 \%)$ de los cuadros diarreicos son producidos por especies de Campylobacter. Sin embargo, aunque el pollo constituye una importante fuente de alimentación humana, no se tiene información sobre la importancia de estas aves como reservorio de
Campylobacter spp. en esta región. Teniendo presente estos antecedentes y considerando que el tipo de habitat y el modo de crianza de estos animales son factores que podrían influir en la proporción de aves portadoras de estas bacterias ${ }^{13.23}$, nos propusimos determinar la frecuencia de aislamiento de Campylobacter spp. en dos poblaciones de pollos criados en distintas condiciones ambientales.

\section{Material y Método}

Fueron estudiadas 200 muestras fecales de pollos obtenidas mediante hisopado cloacal. De ellas, 100 fueron de pollos criados sin confinamiento, pertenecientes a familias de bajo nivel socioeconómico, residentes en la periferia de la ciudad. 
Las 100 restantes fueron de pollos criados en granjas, mantenidos en confinamiento permanente en jaulas de alambre y alimentados con agua potable y raciones comerciales.

Todas las muestras fueron colocadas inmediatamente en el medio de enriquecimiento $0^{10}$, sembradas en placas con Agar Skirrow modificado ${ }^{8} \mathrm{e}$ incubadas a $42^{\circ} \mathrm{C}$ por 48 horas, en microaerofilia.

Las colonias sospechosas fueron identificadas morfologicamente (tinción de Gram con fucsina fenicada como colorante de contraste) y por las pruebas bioquímicas diferenciales propuestas por Goossens y Butzler ${ }^{14}$ y por Lior ${ }^{20}$.

\section{Resultados}

Las especies termotolerantes clásicas de Campylobacter fueron aisladas en el $44,5 \%$ de los 200 pollos estudiados (Tabla 1). En la población de pollos no confinados, Campylobacter spp. fue aislado en el $54,0 \%$, siendo $C$. jejuni la especie más frecuente $(27,0 \%)$. En la población de pollos confinados, la tasa de aislamiento fue menor $(35,0 \%)$, siendo también $C$. jejuni la especie más aislada $(16,0 \%)$. La diferencia global observada entre ambas poblaciones fue estadísticamente significativa $(p<0,05)$.

Los biovars más frecuentes an ambos tipos de aves (Tabla 2), fueron $C$. jejuni biovar I $(29,6 \%$ y $31,4 \%$, respectivamente) y C. coli biovar II $(22,2 \%$ y $17,1 \%$, respectivamente).

Tabla 1 - Aislamiento de las especies termotolerantes de Campylobacter de dos poblaciones de pollos.

\begin{tabular}{lccccc}
\hline Grupo & (n) & C. jejuni & C. coll & C. lari & Total \\
\hline No confinado & 100 & $27_{(27,0)}$ & $17_{(17,0)}$ & $10_{(10,0)}$ & $54_{(54,0) *}$ \\
Confinado & 100 & $16_{(18,0)}$ & $11_{(11,0)}$ & $8_{(8,0)}$ & $35_{(35,0) *}$ \\
\hline Total & 200 & $43_{(21,5)}$ & $28_{(14,0)}$ & $18_{(9,0)}$ & $69_{(44,5)^{*}}$ \\
\hline$*(\%)$ & & & & & \\
$* * 0,05$ & & & & &
\end{tabular}

C. lari fue aislado en el $10 \%$ de las aves no confinadas y en el $8 \%$ de las confinadas, siendo su biovar I el más frecuente.

\section{Discusión}

Las especies termotolerantes de Campylobacter fueron aisladas en los dos grupos de pollos estudiados. En la población no confinada, el 54,0\% fue portadora de estas bacterias. Resultados similares fueron encontrados por Fernández ${ }^{9}(60,0 \%)$, Grados et $a 1 .{ }^{15}(61,4)$, y por Haustein et al. ${ }^{18}(50 \%)$ en pollos criados en condiciones semejantes.

En la población confinada, la frecuencia de aislamiente de Campylobacter spp. fue de $35,0 \%$, valor ligeramente superior al encontrado por Doyle $^{7}(25,2 \%)$ y por Fernández et al. ${ }^{13}(29,4 \%)$ en aves mantenidas en similares condiciones ambientales.

Estos resultados indican que en los pollos que no tienen un confinamiento permanente ni atención sanitaria durante su crianza y que provienen de poblaciones de bajo nivel sanitario, es posible encontrar altos porcentajes de aves portadoras de estas bacterias. Varios autores han sugerido que tanto el sistema de crianza libre como la falta de manejo sanitario adecuado serían factores que condicionaríam a una mayor exposición y riesgo de infección por Campylobacter spp. Esta situación no ocurre en las aves de granja, entre las cuales la frecuencia de muestras positivas fue significativamente menor $(\mathrm{p}<0,05)$. Por su interés económico, estas aves son mantenidas en mejores condiciones higiénico-sanitarias reduciéndose, con ello, las posibilidades de contaminación cruzada ${ }^{13.23}$.

En ambas poblaciones, el biovar más frecuentemente aislado fue $C$. jejuni biovar I, seguido por $C$, coli biovar II.

De las tres especies termotolerantes clásicas, C. jejuni y C. coli son los agentes más comunes de

Tabla 2 - Distribución de los biovars de las especies termotolerantes de Campylobacter aisladas de dos poblaciones de pollos.

\begin{tabular}{|c|c|c|c|c|c|c|c|c|}
\hline \multirow[t]{3}{*}{ Grupo } & \multirow{3}{*}{$\begin{array}{c}N^{0} \text { de } \\
\text { cepas } \\
\text { aisladas }\end{array}$} & \multicolumn{7}{|c|}{ Distribución de Biovars } \\
\hline & & \multicolumn{3}{|c|}{ c. jejuni } & \multicolumn{2}{|c|}{ C. coll } & \multicolumn{2}{|c|}{ C. lari } \\
\hline & & 1 & II & III & I & II & 1 & II \\
\hline $\begin{array}{l}\text { No confinado } \\
\text { Conflnado }\end{array}$ & $\begin{array}{l}54 \\
35\end{array}$ & $\begin{array}{l}16(29,6)^{*} \\
11(31,4)\end{array}$ & $\begin{array}{r}10(18,5) \\
5(14,3)\end{array}$ & $\begin{array}{l}1(1,9) \\
0(0)\end{array}$ & $\begin{array}{l}5(9,3) \\
5(14,3)\end{array}$ & $\begin{array}{r}12(22,2) \\
6(17,1)\end{array}$ & $\begin{array}{l}6(11,1) \\
4(11,4)\end{array}$ & $\begin{array}{l}4(7,4) \\
4(11,4)\end{array}$ \\
\hline
\end{tabular}


enteritis en Latinoamérica, donde $C$. lari ha sido aislado de terneros y aguas servidas en Brasil, de carne de pollo en Costa Rica y de aves acuáticas y de un caso documentado de diarrea humana en Chile". En nuestro trabajo, $C$. lari fue aislado en el $9 \%$ de las muestras, hecho que nos parece importante ya que se estaría aportando evidencias de la participación de los pollos como reservorio de esta bacteria, la cual se encuentra colonizando preferentemente el intestino de gaviotas marinas 3.1 .

La alta frecuencia de aislamiento de Campylobacter spp. $(44,5 \%)$ encontrada en los pollos estudiados demuestra su importancia como reservorio y fuente potencial de infección para el ser humano, ya sea para el personal que tiene constante contacto con ellos en granjas y plantas fae-

\section{Referencias Bibliográficas}

1. ALVAREZ. Y. \& FlORES, J. Poultry as Camplobacter jeftui and Camplobacter coli contamination source. Rel: Lat. Amer. Hicrobiol. 31: 271-3. 1989.

2. BEXIAMIX. J. et al. Description of Campylobacter laridis a nen species comprising the nalidixic acid resistant thermophilic Camprlobacter (XARTC) group. Cum. Herobiol. 8: 231-8. 1983.

3. BLASER. M. I. et al. Reserioirs for human cimprlobacteriosis. f. Hifect. Ois. 141: 665-9. 1980 .

4. BLASER. W. J. et al. Epidemiology of Campylobacter jejunt infections. Epidemiol. Ret.. 5: $15^{-}-76.1983$

5. BLTZLER. J. P. \& SKIRROW. II. B. Campylobacter enteritis. Clin. Gastronterol. 8: $737-65,1979$.

6. DIAS. T. et al. Chicken carcases as a source of Campjlobacter jejuni in Belo Horizonte. Brazil. Ret. Inst. lfed. Trop. S. Patlo. 32: $+1 \dot{4}-$ 8. 1982.

- DOYLE. M. P. Association of Camprlobacter jejthi with laving hens and eggs. Appl. Entiron. Hicrobiol. 47: 533-6. 1984.

8. FER.NAIDEZ. H. Thermophilic species of Camplobacter: bacteriological. epidemiological and pathogenical aspects. S. Paulo, 1983. [Doctoral Thesis - School of Medicine of $S$. Paulo - EPM]

9. FERNANDFZ. H. Species and biorype distribution of thermotolerant campulobacters in animal reservoirs in Southern Chile. Ret'. Inst. .Hed. Trop. S. Paulo. 30: 35-60, 1988.

10. FERXANDEZ. H. Increase of Campllobacter isolation rates using an enrichment medium Ret: Hicrobiol.. 23: 5-7. 1992

11. FERYANDEZ. H. Thermotolerant Campylobacter species associated with human diarrhea in Latin America. Ciêtuc. e Cult. 44: 39-13. 1992. nadoras, o para niños de zonas de precarias condiciones sanitarias donde mantengan crianzas domésticas de estas aves +.23 . Por otro lado, el alto porcentaje de aislamiento encontrado es también um riesgo potencial de contaminación para los consumidores ya que el grado de aislamiento de Campylobacter spp. en el tracto intestinal de las aves parece estar correlacionado con la presencia de estas bacterias en la carne y menudencias después del faenamiento to

Pensamos que deben realizarse mayores estudios relacionados con los aspectos ecológicos de estas bacterias, para contribuir a clarificar la epidemiología de la campylobacteriosis, la cual parece ser un fenómeno muy complejo en los países subdesarrollados.

12. FERXANDF7. H. et. al Camplobacter laridis: first clinical isolation and identification of reservoir in Chile. Rez'. léd. Chile, 118: 699. -01. 1990.

13. FERNANDEZ. H. et al. Ocurrence of themotolerant species of Campllobacter in three groups of hens maintained under different environmental conditions. Ret. Hicrobiol. 24: 265-8, 1993.

14. GOOSSEIS, H. \& BLTZLER, T. P. Isolation and identification of Campllobacter spp. In: vachamkin. I. et al.. ed. Campulobacter jejnni: current status and futhre trends. Washington, DC, American Society for Microbiology. 1992. p. 93-109.

15. GRADOS. O, et al. Paediatric Campyobacter. diarrioea from houschold exposure to live chickens in Lima. Peru. Bull. Wonld Health Organ. 66: 369-74. 1988.

16. GRANT, I. H. et al. Broiler chickens as potential source of campllobacter infections in humans. f. Chin . Hicrobiol. 11: 508-10. 1980.

$1^{-}$. HARRIS, N.V. et al. The role of poultry and meats in the etiology of Campliobacter jejunicoli enteritis. Am. J. Public. Health. 76: i0 $-11,1986$.

18. HALSTEIN. A. T. et al. Serage gronn Lema as a protein source for chickens: final report to AID Washington, D.C. of project 3. F23. Washington. 198 ?

19. IEVIX, 5. \& GOODMAN. L. Campylobacter infections today. JAWA. 253: 1303. 1985.

20. LIOR. H. Nen: extende biotyping scheme for Camplobacter jejui. Camplobacter colli and "Campllobacter laridis". J. Clin. Hicrobiol. 20: 636-40, 1984.

21. PRESCOTT. I.F. \& MLTROE, D.L. Camplobacter jejumi in man and domestic animals. I. Am. let. hed. Ass., 181: 1524-30. 1982. 
22. SAKUMA, H. et al. Occurrence of Campylobacter jejuni and Campylobacter coli in retail raw chicken meat and giblets in Sào Paulo, Brazil. Rev. Microbiol., 23: 13-6, 1992.

23. SHANE, S. M. The significance of Campylobacter jejuni infection in poultry: a review, Avian Patbol, 21: 189-213, 1992.

24. WEMPE, J.M. et al. Prevalence of Campylobacter jejuni in two California chicken processing plants. Appl. Environ., 45: 355-9, 1983.

\begin{abstract}
The isolation rates of thermotolerant Campylobacter species in free-ranging domestic chickens and confined chickens from Iquitos city, Pen, were determined. Campylobacter spp. were isolated in $54,0 \%$ of the former group of chickens, being less frequent $(35,0 \%)$ in the latter $(\rho<0,05)$. Of the classical thermotolerant species, $\mathrm{C}$. jejuni and $\mathrm{C}$. coli were the most frequent. However, the presence $\mathrm{C}$. lar! suggests that the chickens might be an important reservoir of this bacterium.
\end{abstract}

Campylobacter, isolation chickens, microbiology. Disease reservoirs.

\title{
Resumo
}

Estudou-se a freqüencia de isolamento de Campylobacter spp. em frangos domésticos e frangos mantidos em continamento permanente, na cidade de lquitos (Peru). Campylobacter spp. foi isolado em 54,0\% no primeiro gnipo e $35,0 \%$ no segundo $(p<0,005)$. Das especies termotolerantes clássicas, as mals frequentes foram $C$. jejuni $\theta \mathrm{C}$. coli. $A$ presença de C. lari nessas aves, assinala a importáncia delas como reservatório natural desse microrganismo.

Campylobacter, isolamento e purificaçâo. Galinhas, microbiologia. Reservatórios de doenças. 